\title{
WAyPoint Flight PARAMETER Comparison of AN AUTONOMOUS UAV
}

\author{
Nils Gageik $^{1}$, Michael Strohmeier $^{2}$ and Sergio Montenegro ${ }^{3}$ \\ ${ }^{1}$ Chair of Computer Science 8, University of Würzburg, Germany \\ gageikeinformatik . uni-wuerzburg.de \\ ${ }^{2}$ Chair of Computer Science 8, University of Würzburg, Germany \\ michael.strohmeier@stud-mail.uni-wuerzburg.de \\ ${ }^{3}$ Chair of Computer Science 8, University of Würzburg, Germany, \\ sergio.montenegro@uni-wuerzburg.de
}

\begin{abstract}
The present paper compares the effect of different waypoint parameters on the flight performance of a special autonomous indoor UAV (unmanned aerial vehicle) fusing ultrasonic, inertial, pressure and optical sensors for $3 D$ positioning and controlling. The investigated parameters are the acceptance threshold for reaching a waypoint as well as the maximal waypoint step size or block size. The effect of these parameters on the flight time and accuracy of the flight path is investigated. Therefore the paper addresses how the acceptance threshold and step size influence the speed and accuracy of the autonomous flight and thus influence the performance of the presented autonomous quadrocopter under real indoor navigation circumstances. Furthermore the paper demonstrates a drawback of the standard potential field method for navigation of such autonomous quadrocopters and points to an improvement.
\end{abstract}

\section{KEYWORDS}

Autonomous UAV, Quadrocopter, Quadrotor, Waypoint Parameter, Navigation

\section{INTRODUCTION}

Despite the intensive research efforts of the past few years autonomous UAVs and especially autonomous quadrocopters are still a topic of interest. This is due to the complexity of the problem which has several drawbacks, such as price and dependency on external devices or certain environmental circumstances (e.g. good lighting conditions).

Autonomous outdoor quadrocopters using GPS for positioning and navigation are commercially available and well researched [1-4], but in GPS-denied environments such systems fail. Impressive are autonomous systems, which use optical tracking cameras for aggressive manoeuvres, such as flying through small windows [5]. This becomes possible because of the very accurate external optical tracking system and is not applicable in unknown or changing environments. Because of the dependency on external systems, these two solutions are more suitably called semi-autonomous.

For fully autonomous systems a number of different approaches already exist, which still suffer from their own drawbacks. There exist very simple commercially available quadrocopters with four infrared sensors for collision avoidance, which neither covers the full environment nor allows for autonomous navigation [3, 6]. Other Systems, which overcome such drawbacks, use 
laser scanners [7, 8], stereo cameras or the Kinect camera from Microsoft [9]. Those approaches are complex, demand a high computational burden and are often executed on external processors. Furthermore laser scanners are very expensive while the Kinect camera is quite big for a small UAV. In comparison, standard video cameras are still much cheaper and smaller.

For a low-cost, quick, accurate, reliable and simple solution, the AQopterI8 (Figure 1), an autonomous indoor quadrocopter, was developed, which is used here for comparing waypoint flight parameters. The quadrocopter fuses one inertial, one ultrasonic, two infrared and one pressure sensor to estimate its height over the ground [10]. An optical mouse sensor is used to compute the position in the $\mathrm{x}-\mathrm{y}$-plane. Hence, a fully autonomous 3D pathway flight is possible.

All data processing is performed on the UAV. An external computer with a commanding interface is for set-up purposes only. The presented system is part of the AQopterI8 project, which aims to develop an autonomous flying quadrocopter for indoor application, where navigation in unknown and changing environments is required. The system for collision avoidance is not discussed here and can be found in a different work [11].

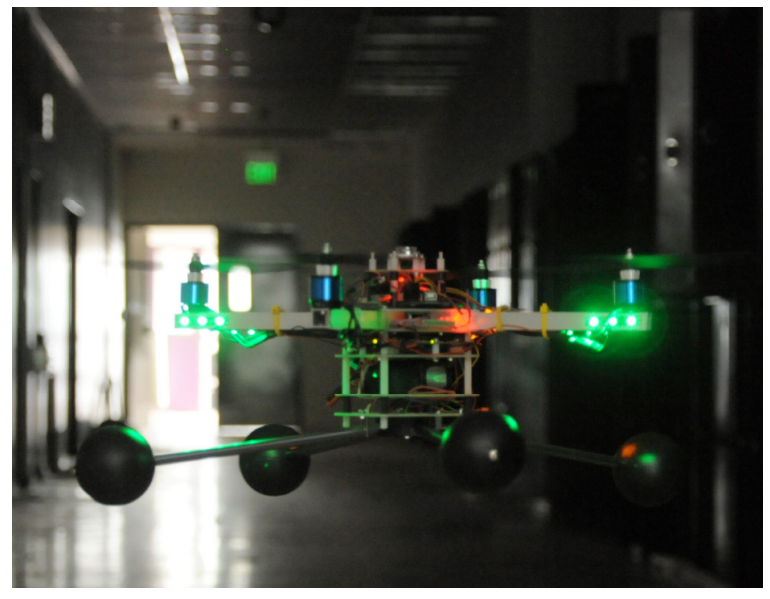

Figure 1. AQopterI8

Beside the sensor and controller performance, the accuracy and elapsed time of a pathway flight is determined by the acceptance threshold of a waypoint and the maximum step size between two sequencing waypoints.

The acceptance threshold is the radius around a waypoint, which still belongs to a waypoint. The maximum step size is the maximum distance between two executed waypoints. More waypoints on the same linear track may result in more accuracy, but may also lead to more delay, since every waypoint has to be checked off one after another. These two parameters and their effect on the performance (time, accuracy) of the system - an autonomous quadrocopter with on-board sensors - are investigated here.

This comparison has been performed empirically by experiments with a real system, since investigation by simulation seemed not worthwhile because of the complexity of the system and the many unknown, difficult to determine or unpredictable effects such as (small) orientation errors from vibrations and yaw orientation drift, wind reflections from walls or the ground and height errors due to height sensor errors and a changing voltage level of the battery. In particular changing lighting and non-optimal surface conditions as well as attitude and altitude changes of the flying quadrocopters make it difficult to quantify errors on the optical position sensor. However the presented results can help to parameterize and revise a simulation. 
International Journal of Artificial Intelligence \& Applications (IJAIA), Vol. 4, No.3, May 2013

\section{TERM DEFINITION}

As already mentioned, the acceptance threshold $t$ is defined as the radius around a waypoint $\mathrm{W}(\mathrm{x}, \mathrm{y})$ [Figure 2 left]. When the quadrocopter reaches this area, the waypoint is supposed to be reached and it is switched to the next waypoint. The parameter $d_{\max }$, the maximum distance between two waypoints, determines the number of waypoints needed to fly a fixed pathway. As an example Figure 2 (right) shows two times the same (simple) pathway with $d_{\max }=1 \mathrm{~m}$ and $d_{\max }$ $=0.1 \mathrm{~m}$.

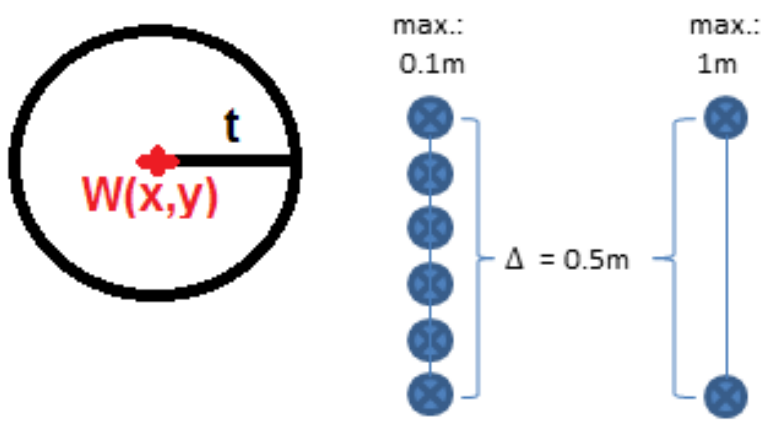

Figure 2. Acceptance threshold t (left); Two pathways with $d_{\max }=0.1 \mathrm{~m}$ and $d_{\max }=1 \mathrm{~m}$ (right)

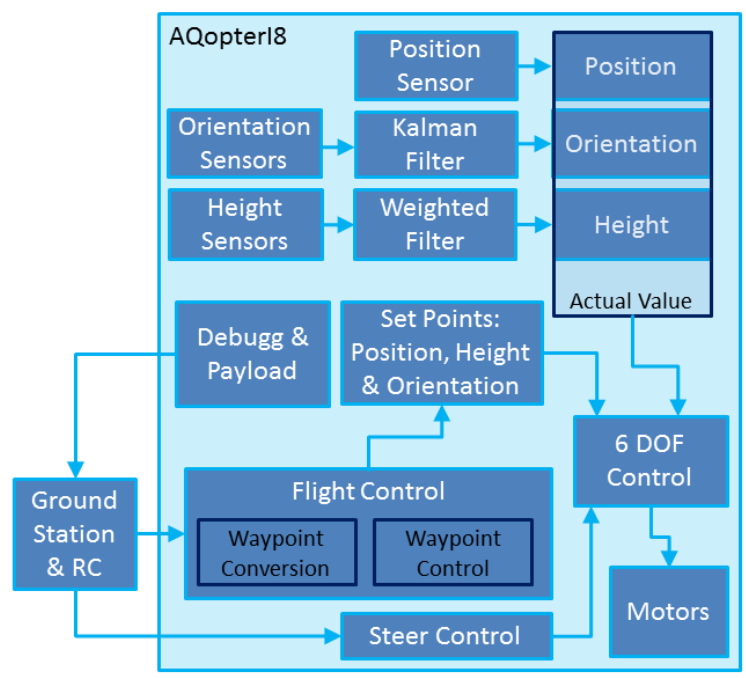

Figure 3. System Concept

\section{CONCEPT}

\subsection{General System Overview}

Figure 3 demonstrates the concept of the system. The quadrocopter has all the sensors necessary for positioning and orientation on-board. The 2D-position is directly computed from the optical position sensor (optical mouse sensor), while the height over ground is computed using a weighted filter [Gageik, 2012b]. This filter performs a data fusion of ultrasonic, infrared, inertial and pressure sensors. The orientation in roll-pitch-yaw and quaternion is computed using a Kalman Filter fusing a 3 DOF (degree of freedom) accelerometer with a 3 DOF gyroscope. 
International Journal of Artificial Intelligence \& Applications (IJAIA), Vol. 4, No.3, May 2013

Before operation, a list of waypoints is sent remotely from the ground station to the quadrocopter, which is the minimum amount of waypoints for a pathway flight set up by the operator. The quadrocopter then transforms this waypoint list according to $\mathrm{d}_{\max }$ (waypoint conversion) and determines the set points for the 6 DOF controller. The 6 DOF control consists of 6 PID Controllers, one for every DOF. The second input of the 6 DOF control module is constituted by the actual values of position, orientation and height, which are determined by the according sensors and data fusion principles.

\subsection{Flight Control}

The Flight Control consists of the Waypoint Conversion and the Waypoint Control. The Waypoint Conversion is processed during the flight. Whenever the flight to a new predefined waypoint $W(j+1)==\left\langle W_{x}(j+1), W_{y}(j+1)>\right.$ is executed, it is proved, if the distance of the waypoint on one axis is greater than $d_{\max }$. Here $j$ determines the Waypoint index. If necessary waypoints are added in between, to achieve a distance between any waypoint, that is not greater than $d_{\max }$. The number of added waypoints I and their position $\mathrm{P}_{\mathrm{ji}}$ are determined by formula 1 . The index $\mathrm{i}$ is to distinguish added waypoints between every original waypoint $\mathrm{W}(\mathrm{j})$.

$$
\begin{gathered}
\Delta W_{x}=W_{x}(j+1)-W_{x}(j) \\
\Delta W_{\mathrm{y}}=W_{y}(j+1)-W_{y}(j) \\
\left.\Delta=\max \left(\left|\Delta W_{x}\right|,\left|\Delta W_{y}\right|\right)\right) \\
\mathrm{I}=\left\lceil\frac{\Delta}{d_{\max }}\right\rceil \\
\Delta x=\frac{\Delta W_{x}}{I} \\
\Delta y=\frac{\Delta W_{\mathrm{y}}}{I} \\
\mathrm{P}_{\mathrm{ji}}=\left\langle\mathrm{W}_{\mathrm{x}}(\mathrm{j})+\Delta x \cdot \mathrm{i}, \mathrm{W}_{\mathrm{y}}(\mathrm{j})+\Delta y \cdot \mathrm{i}>\right.
\end{gathered}
$$

The waypoint control proves, if the current waypoint is reached and if so, it switches to the next waypoint.

\section{IMPLEMENTATION}

\subsection{Hardware Design}

The hardware-design of the quadrocopter is depicted in Figure 4 (left). The ADNS-3080 (optical mouse sensor) [13], the SRF-02 (ultrasonic distance sensor), the GP2Y0A710K0F (infrared long distance sensor) and the GP2Y0A02YK (infrared medium distance sensor) are mounted on the bottom plate (Figure 4, right). The ADNS is the position sensor and the ultrasonic and infrared sensors, together with the BMP085 (pressure sensor) and the IMU3000 (inertial measurement unit), are the height sensors according to figure 3. Two infrared sensors are used to cover all distances between $30 \mathrm{~cm}$ and $500 \mathrm{~cm}$, which is not possible with only one infrared sensor. The same IMU is also used for orientation computation (orientation sensor). 
International Journal of Artificial Intelligence \& Applications (IJAIA), Vol. 4, No.3, May 2013
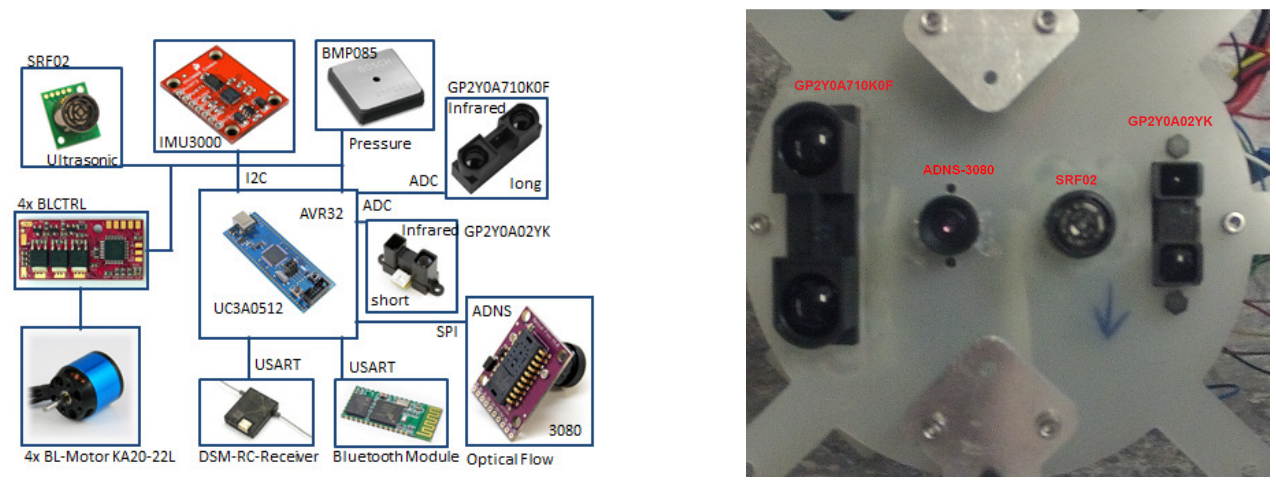

Figure 4. Hardware Design (left), Bottom-Plate (right)
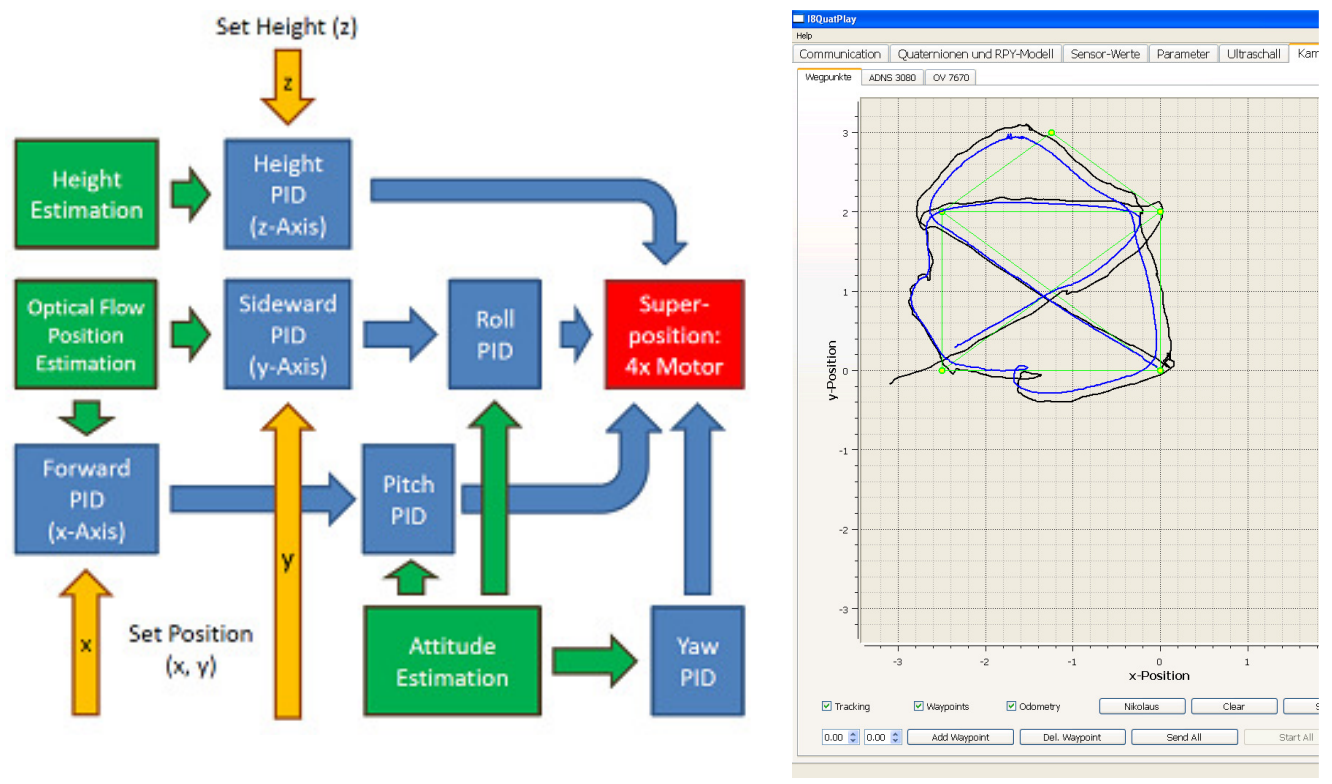

Figure 5. Control Design (left): Sensors \& Signal Processing (Green), Commands \& External Set Points (Yellow), Internal Control Data Processing (Blue), Superposition \& Output (Red)

Qt Control Software (right)

\subsection{Control Design}

For each degree of freedom an empiric optimized PID controller was implemented. For a 6 DOF system this amounts to 6 cascading PID controllers (Figure 5, left). The sample time of the system is $10 \mathrm{~ms}$.

The inputs of the position controllers [12] comprise the waypoints $\mathrm{P}_{\mathrm{ji}}$ and the optical flow measurements. The optical flow measurements correspond to the position. The units of the position PID inputs are represented in optical flow position data, which are constituted by sensor raw data integration. The position is calibrated by the initial offset. The outputs of the position controllers are the set points for roll and pitch in degree. In order to increase the stability of the two position controllers as well as to limit the overshoot, the total sum of the P- and D-term is limited to $5^{\circ}$. The D-gain has a value of 1.75 and cannot be raised much further, since this would 
International Journal of Artificial Intelligence \& Applications (IJAIA), Vol. 4, No.3, May 2013

lead to an unstable (oscillating) system. A small average filter over the delta error improved the performance. A longer average filter improves the stationary behaviour, but worsens the dynamic response to a new set point.

The P-gain has a value of 0.0125 . This value optimizes the step response and overshoot. A significantly lower P-gain can reduce the overshoot, but also inhibits reaching the set point. The Integral-gain has a value of 0.00003 . The system reacts in a very sensitive way on a higher I-gain, as this reduces the stability and increases the overshoot, leading to oscillation. To reduce the unwanted effects of the I-term, two integration thresholds are used. If the magnitude of the Dterm is greater than 0.4 or the magnitude of the position error is greater than $1 \mathrm{~m}$, the error is not integrated. This derives from the idea that the I-term should not be accumulated, while the quadrocopter is approaching a new waypoint, but only if it is not reaching the waypoint (stationary error).

\subsection{Control Software}

For debugging and evaluation purposes as well as to control the quadrocopter a control program (Figure 5, right) was developed using Qt [15]. The control software communicates with the quadrocopter using bluetooth and is used to send the waypoints $\mathrm{W}_{\mathrm{j}}$ to the quadrocopter during the set-up phase and to start the mission. Also it is used to receive and process the evaluation data and other debugging information. Thus the performance of the flight, determined by the accuracy and time, can be quantified. All other functionality such as controlling starting, flying the waypointlist and landing is performed by the on-Board AVR microcontroller.

The time $t_{\mathrm{e}}$ is defined as the elapsed time between flying from the first waypoint to the last waypoint. The quadrocopter sends a signal after each waypoint is reached. After receiving the last signal, the control software computes the time of the pathway flight.

The accuracy $\mathrm{A}$ is defined as the average distance between the closest point on the current pathway and the current position of the quadrocopter. The accuracy is also computed by the control software after the experiment. This is done by comparing every measured position with a discrete list of waypoints of the current pathway. There is one dedicated list of waypoints for every two Waypoints $\mathrm{W}_{\mathrm{j}-1}$ and $\mathrm{W}_{\mathrm{j}}$. This list is generated by adding a waypoint after every $1 \mathrm{~cm}$ from the last valid waypoint $\mathrm{W}_{\mathrm{j}-1}$, until the current valid original waypoint $\mathrm{W}_{\mathrm{j}}$ is reached. The communication and evaluation data update rate is $100 \mathrm{~ms}$. This means that a measurement is taken and transferred to the ground station 10 times a second.

\section{Evaluation}

In every experiment the same list of waypoints $\mathrm{W}_{\mathrm{j}}$ (Figure 6) was sent to the quadrocopter and its position was tracked with the optical tracking system PPT X4 from WorldViz [WorldViz] to evaluate the performance of the autonomous flight. The accuracy A and time $t_{\mathrm{e}}$ were determined after the experiment using the control software.

\subsection{Preliminary Tests}

To find the range of the optimal and most interesting parameters, first several preliminary runs with wider bandwidth of parameter range were performed. This was also necessary, because one experiment lasts from one to five minutes, and with one battery about two or three experiments could be performed properly, avoiding too much influence of the battery level on the results of the experiment. 
International Journal of Artificial Intelligence \& Applications (IJAIA), Vol. 4, No.3, May 2013

The threshold $\mathrm{t}$ was investigated in the range of $10 \mathrm{~cm}$ to $25 \mathrm{~cm}$. The experiments clearly showed, that $10 \mathrm{~cm}$ is too low, resulting in a big delay. The time $t_{\mathrm{e}}$ increased dramatically, especially with a higher number of waypoints and lower $\mathrm{d}_{\max }$. Since the standard deviation of the position error on position hold is already about $10 \mathrm{~cm}$ [12], the system is too dynamic for such a low threshold.

For $\mathrm{d}_{\max }$ values between $1 \mathrm{~cm}$ and $300 \mathrm{~cm}$ were investigated. Contrary to expectations, values between $10 \mathrm{~cm}$ and $50 \mathrm{~cm}$ performed very poorly. Such low values do not increase the accuracy, but rather the time. Especially in combination with a low value for $t$, the flight sometimes lasted till the battery was empty.

The experiment clearly highlighted the problem. More waypoints mean more set point jumps and more controller error jumps, leading to a less smooth flight. These jumps lead to observable pitch or roll movements of the quadrocopter during one linear path and the flight becomes jumpy. As pitch and roll movements lead to wrong optical flow measurements of the position sensor, these jumps intensify the problem. These jumps can be seen as position jump measurements of the ADNS on the flight map of the control software (Figure 9). This problem will later be referred to as the jump effect.

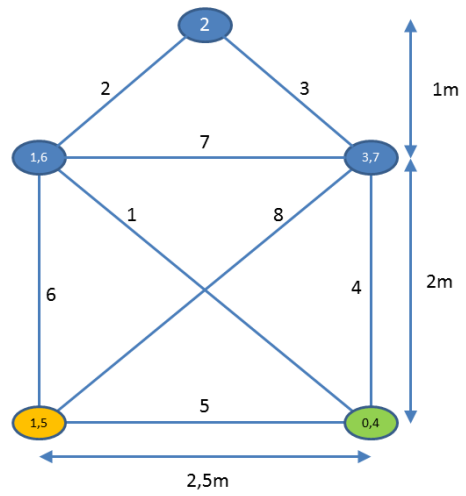

Figure 6. Left: Waypoint order with pathway (total 18.6m) for autonomous flight from starting (green) till landing (yellow)

\subsection{Final Experiments}

Therefore, the best results were attained with $t$ in the range of $50 \mathrm{~cm}$ and $300 \mathrm{~cm}$ and $\mathrm{d}_{\max }$ between $15 \mathrm{~cm}$ and $25 \mathrm{~cm}$. Every one of such 9 possible combinations was repeated 5 times. Table 1 shows the results quantified in time and accuracy. Some experiments, marked with "x", could not be finished, because the quadrocopter failed to find one of the waypoints in an acceptable time.

Even though the variance of the results within the same parameter category is, for some combinations, relatively high (Table 1), which underlines the dependency on many effects, the complexity of the system and the difficulty of a proper simulation because of the multiple mentioned unpredictable noises, the results nevertheless provide interesting and clear answers. As can be derived from the control design and therefore as expected, a higher threshold $t$ and a higher step size dedicated by $d_{\max }$ result in a faster flight, indicated by a lower elapsed time $t_{e}$ (Table 2). Nevertheless it was also shown, that more waypoints do not mean more accuracy. Even with a $d_{\max }$ of $1 \mathrm{~cm}$ (Table 1), leading to a three times longer flight time compared to $\mathrm{d}_{\max }=1 \mathrm{~m}$, the accuracy was still in the same range.

Furthermore, a lower threshold t only increases the accuracy to a certain limit (Table 3). This is because too low values, meaning high accurate positioning, are not performable due to the dynamic of the system. A lower threshold $t$ even decreases the accuracy, as waypoints are dropped and the quadrocopter needs to fly back. 
International Journal of Artificial Intelligence \& Applications (IJAIA), Vol. 4, No.3, May 2013

Table 1. Elapsed time $\mathrm{t}_{\mathrm{e}}[\mathrm{s}]$, and accuracy A [m] and their Means over 40 evaluated autonomous pathway flights

\begin{tabular}{|c|c|c|c|c|c|c|c|c|c|c|c|}
\hline $\mathbf{d}_{\max }[\mathbf{m}]$ & $\mathbf{t}[\mathbf{c m}]$ & \multicolumn{4}{|c|}{$\mathbf{t}_{\mathrm{A}}[\mathbf{s}]$} & \multicolumn{5}{|c|}{$\mathbf{A}[\mathbf{m}]$} \\
\hline \multirow{3}{*}{3} & 15 & 43 & 65.4 & 41.4 & 46.3 & 42.4 & 0.27 & 0.29 & 0.24 & 0.28 & 0.29 \\
\cline { 2 - 13 } & 20 & 41.5 & 39.6 & 41.2 & 40.3 & 40.5 & 0.17 & 0.18 & 0.29 & 0.35 & 0.3 \\
\cline { 2 - 13 } & 25 & 36.3 & 40.5 & 36.9 & 40.6 & 34.3 & 0.53 & 0.27 & 0.32 & 0.17 & 0.22 \\
\hline \multirow{3}{*}{1} & 15 & 80.8 & 83.8 & 91.4 & 75.7 & 120 & 0.44 & 0.34 & 0.32 & 0.24 & 0.31 \\
\cline { 2 - 12 } & 20 & 75.9 & 50.5 & 53.5 & 70.8 & 53.6 & 0.18 & 0.14 & 0.13 & 0.3 & 0.25 \\
\cline { 2 - 12 } & 25 & 66.6 & 49 & 48.6 & 49.3 & 46.8 & 0.36 & 0.18 & 0.16 & 0.25 & 0.25 \\
\hline \multirow{3}{*}{0.5} & 15 & 155 & 127 & 110 & 125 & 116 & 0.47 & 0.19 & 0.23 & 0.3 & 0.13 \\
\cline { 2 - 12 } & 20 & $\mathrm{x}$ & 92.4 & $\mathrm{x}$ & - & - & $\mathrm{x}$ & 0.18 & $\mathrm{X}$ & - & - \\
\cline { 2 - 12 } & 25 & $\mathrm{x}$ & $\mathrm{x}$ & - & - & - & $\mathrm{x}$ & $\mathrm{x}$ & - & - & - \\
\hline 0.01 & 25 & 144 & 143 & - & - & - & 0.2 & 0.23 & - & - & - \\
\hline
\end{tabular}

Table 2. Mean of elapsed time $\mathrm{t}_{\mathrm{e}}[\mathrm{s}]$ and accuracy A [m]

\begin{tabular}{|c|c|c|c|c|}
\hline \multirow{2}{*}{ Mean } & $\mathbf{d}_{\max }[\mathbf{m}]$ & \multicolumn{3}{|c|}{$\mathbf{t}[\mathbf{c m}]$} \\
\cline { 2 - 5 } & & $\mathbf{1 5}$ & $\mathbf{2 0}$ & $\mathbf{2 5}$ \\
\hline \multirow{3}{*}{$t_{\mathbf{e}}[\mathbf{s}]$} & 3 & 47.7 & 40.62 & 37.72 \\
\cline { 2 - 5 } & 1 & 90.34 & 60.82 & 52.6 \\
\cline { 2 - 5 } & 0.5 & 126.46 & $\mathrm{x}$ & $\mathrm{X}$ \\
\hline \multirow{3}{*}{$\mathbf{A}[\mathbf{m}]$} & 3 & 0.274 & 0.258 & 0.302 \\
\cline { 2 - 5 } & 1 & 0.33 & 0.2 & 0.24 \\
\cline { 2 - 5 } & 0.5 & 0.264 & $\mathrm{x}$ & $\mathrm{x}$ \\
\hline
\end{tabular}

Figure 7 shows the performance of some flights with different parameters. The general effect of these parameters on the flight performance is clearly shown. With a high $d_{\max }$ of $3 \mathrm{~m}$ and a relatively high $t$ of $25 \mathrm{~cm}$, the flight is performed fast and in straight lines. For run \#1, of which the pathway is shown in the upper left corner of figure 9 (Flight A), it lasted 36,3s with an accuracy of $0.53 \mathrm{~m}$. The lower $\mathrm{d}_{\max }$, the more uneven the flight, which only looks worse to human eyes, but accuracy is generally slightly better for $d_{\max }=1 \mathrm{~m}, d_{\max }=0.5 \mathrm{~m}$ and $d_{\max }=1 \mathrm{~cm}$ compared to $d_{\max }=3 \mathrm{~m}$. This is because the quadrocopter flies closer to the pathway line, in case there are more waypoints. This benefit is reduced by the jump effect and the fact that the quadrocopter can drop waypoints and then has to fly back. These turns can also be seen in figure 9, especially in the bottom right corner (flight D). This flight lasted 144 seconds with an accuracy of $0.2 \mathrm{~m}$. Though the flight time of Flight D was about 4 times longer compared to the Flight A, the accuracy was two times better.

There exists an initial fix starting offset between optical tracking and optical position sensor, because of the starting procedure, which is not compensated in figure 7 but in the computation of the accuracy. 


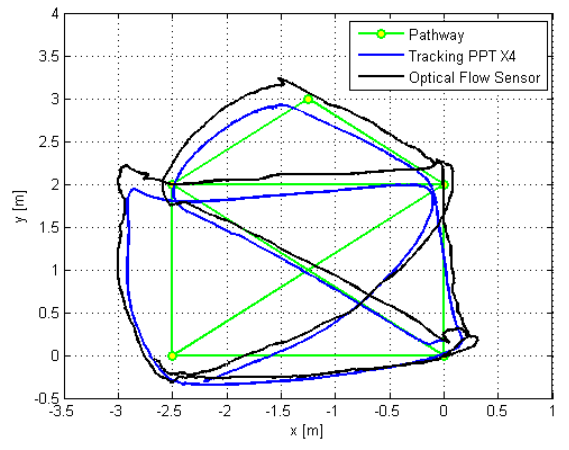

A: $d_{\max }=3 \mathrm{~m}, t=25 \mathrm{~cm}(\# 1)$

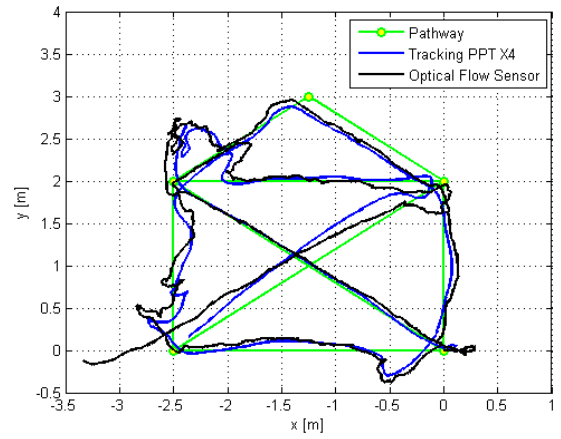

$C: d_{\max }=50 \mathrm{~cm}, t=15 \mathrm{~cm}(\# 4)$

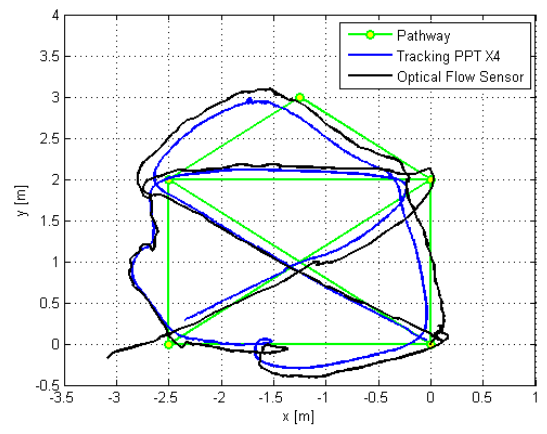

$B: d_{\max }=1 \mathrm{~m}, t=20 \mathrm{~cm}(\# 2)$

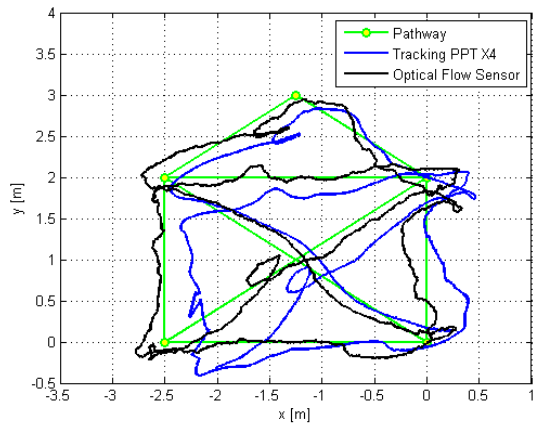

$D: d_{\max }=1 \mathrm{~cm}, t=25 \mathrm{~cm}(\# 1)$

Figure 7. Four pathway flights (pathway green) tracked with the PPT X4 (blue) and the on-board optical flow sensor (black)

\section{Conclusion And Perspective}

This paper demonstrates the effect of the acceptance threshold $t$ and the amount of waypoints determined by the maximum distance between two waypoints $d_{\max }$ on the flight performance of an autonomous UAV. The results were quantified by the elapsed time $t_{e}$ and accuracy $A$ of an autonomous waypoint flight. In spite of our expectations, having more waypoints, instead of increasing the accuracy, reduces it in many cases or has no detectable positive effect. One reason for this is the already mentioned jump effect.

This is also the case for a threshold $t$ that is too low, in which case the quadrocopter has problems reaching the waypoints and tends to drop waypoints. If so, it has to fly back. This reduces the accuracy.

The double standard deviation of the system on position hold is about $16 \mathrm{~cm}$. At the same time this can be seen to be about the lowest limit for $t$, as the system performed very badly for $\mathrm{t}=10 \mathrm{~cm}$ and also not well with $\mathrm{t}=15 \mathrm{~cm}$ compared to $\mathrm{t}=20 \mathrm{~cm}$.

The optimal parameters for a trade-off between time and accuracy are $t=20 \mathrm{~cm}$ and $\mathrm{d}_{\max }=1 \mathrm{~m}$. The average speed of this set up for the complete $18 \mathrm{~m}$ pathway (figure 8 ) is about $0.3 \mathrm{~m} / \mathrm{s}$, which is very much limited by the sensor and control performance.

A $d_{\max }$ of $0.5 \mathrm{~m}$ or lower is not suitable according to the results obtained in our experiments. Furthermore, for a time optimal flight, $\mathrm{d}_{\max }$ should be set to infinity. This result contradicts standard potential field methods for navigation and collision avoidance, where the next set point 
is the nearest free block on the way. In this case the quadrocopter would make many short steps, which would lead to the jump effect problem and wrong optical flow measurements. Instead a method is suggested, which uses a minimum amount of set point changes and large set point changes.

The pathway flight performance, especially the accuracy and the speed, may be further improved with changes in the sensor and control concept, as well as the waypoint acceptance procedure. To overcome the jump effect we implemented a bottom plate (Figure 8), which is steered by two servos to keep its orientation in the x-y-plane. Initial experiments showed that, although it does not improve the stationary behaviour on position hold, it can slightly reduce the jump effect and improve accuracy, but not totally solve this problem. A further parameter optimization and deeper investigation of the potential of this approach is necessary; however this is a different topic. The new bottom plate may also allow more dynamic movements, which are not possible right now, because of the bad effect of roll and pitch rotations on the measurements of the position sensor.

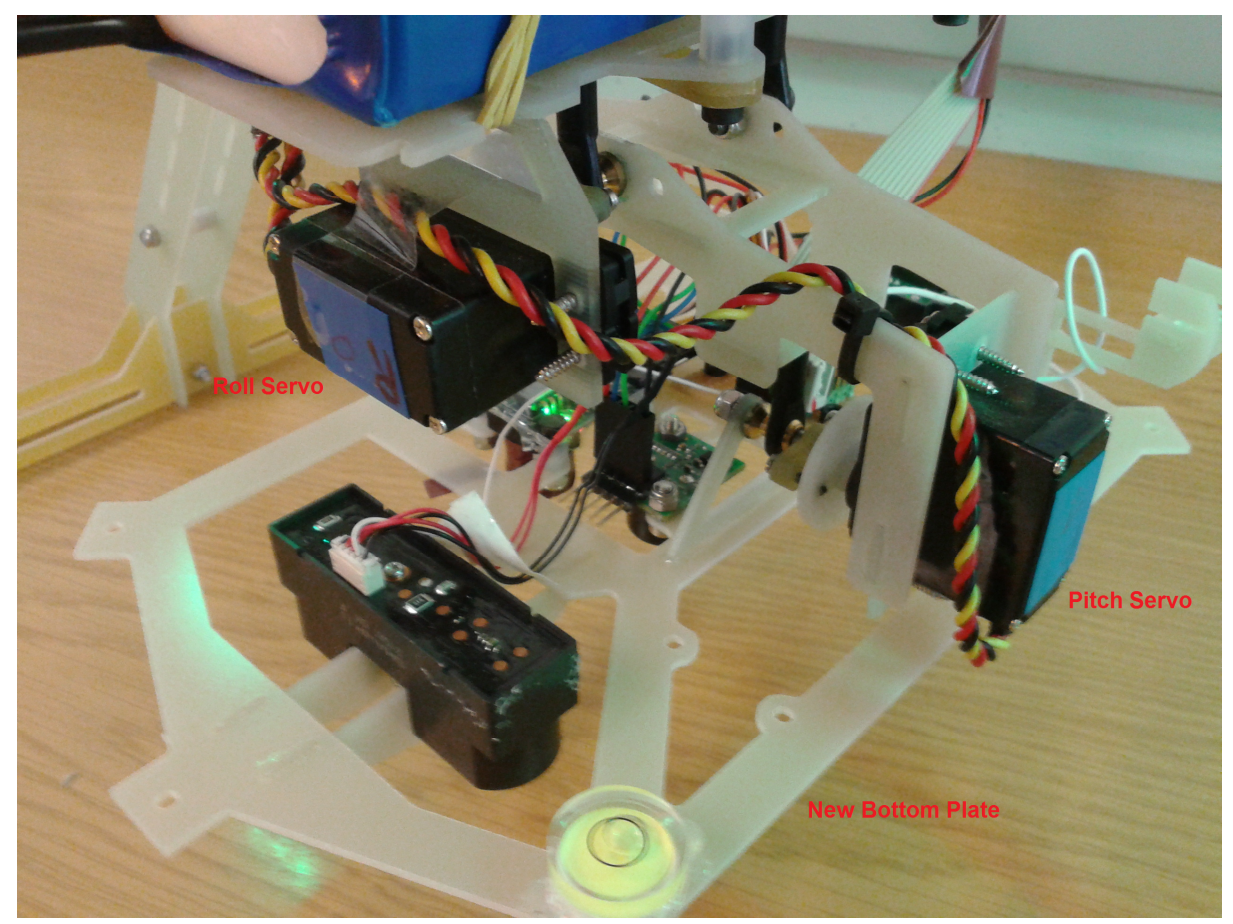

Figure 8. Bottom-Plate with Servo-Motors

\section{ACKNOWLEDGEMENTS}

The author would like to thank Barbara Tabisz for reviewing this paper. This publication was funded by the German Research Foundation (DFG) and the University of Wuerzburg in the funding program Open Access Publishing.

\section{REFERENCES}

[1] Nonami K.(2010), Autonomous Flying Robots, Springer, ISBN-10: 4431538550

[2] Microdrones GmbH, www.microdrones.com

[3] ArduCopter, http://code.google.com/p/arducopter

[4] HiSystems GmbH, www.mikrokopter.de 
International Journal of Artificial Intelligence \& Applications (IJAIA), Vol. 4, No.3, May 2013

[5] Mellinger D. et al, Trajectory Generation and Control for Precise Aggressive Maneuvers with Quadrotors, The International Journal of Robotics Research, April 2012, Vol. 31 No.5

[6] Walkera, http://www.walkera.com/en/

[7] Grzonka S. et al, A Fully Autonomous Indoor Quadrotor, IEEE Transactions on Robotics, Vol. 28 No. 1, Februrary 2012

[8] Gronzka S., Mapping, State Estimation, and Navigation for Quadrotors and Human-Worn Sensor Systems, PhD Thesis, Uni Freiburg, 2011

[9] Lange S., Sünderhauf N. Neubert P., Drews S., Protzel P., Autonomous Corridor Flight of a UAV Using a Low-Cost and Light-Weight RGB-D Camera, Advances in Autonomous Mini Robots, 2012, ISBN: 978-3-642-27481-7

[10] Gageik N., Rothe J., Montenegro S., Data Fusion Principles for Height Control and Autonomous Landing of a Quadrocopter, UAVveek 2012

[11] Gageik N., Mueller T., Montenegro S., Obstacle Detection and Collision Avoidance Using Ultrasonic Distance Sensors for an Autonomous Quadrocopter, UAVveek 2012

[12] Strohmeier M., Implementierung und Evaluierung einer Positionsregelung unter Verwendung des optischen Flusses, Würzburg 2012, BA Thesis

[13] ADNS-3080 High-Performance Optical Mouse Sensor, Data Sheet, Avago Technologies, http://www.avagotech.com

[14] WorldViz, www.worldviz.com

[15] Qt Project, http://qt.digia.com

\section{AUTHORS}

Dipl.-Ing. Nils Gageik is working as a research assistant and $\mathrm{PhD}$ student at the Chair Aerospace Information Technology at the University of Wuerzburg. He received his diploma from the RWTH Aachen University 2010 in Computer Engineering

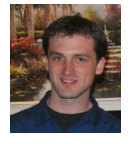

B. Sc. Michael Strohmeier is a Master Student in the international spacemaster program. He received his Bachelor 2012 at the University of Wuerzburg.

Prof. Dr. Sergio Montenegro is holder of the Chair Aerospace Information Technology at the University of Wuerzburg.

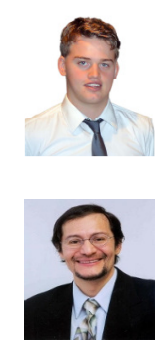

